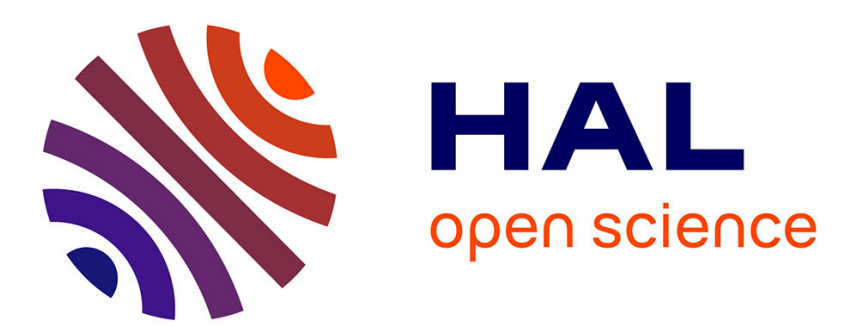

\title{
La fiche de lecture ou la bureaucratisation d'une technique d'animation culturelle
}

\author{
Jean-Marie Privat, Marie-Christine Vinson
}

\section{To cite this version:}

Jean-Marie Privat, Marie-Christine Vinson. La fiche de lecture ou la bureaucratisation d'une technique d'animation culturelle. Pratiques: linguistique, littérature, didactique, 1996, 90, pp.83-94. 10.3406/prati.1996.1775 . hal-03180263

\section{HAL Id: hal-03180263 \\ https://hal.univ-lorraine.fr/hal-03180263}

Submitted on 24 Mar 2021

HAL is a multi-disciplinary open access archive for the deposit and dissemination of scientific research documents, whether they are published or not. The documents may come from teaching and research institutions in France or abroad, or from public or private research centers.
L'archive ouverte pluridisciplinaire HAL, est destinée au dépôt et à la diffusion de documents scientifiques de niveau recherche, publiés ou non, émanant des établissements d'enseignement et de recherche français ou étrangers, des laboratoires publics ou privés. 


\title{
La fiche de lecture ou la bureaucratisation d'une technique
} d'animation culturelle

\author{
Jean-Marie Privat, Marie-Christine Vinson
}

\section{Résumé}

L'histoire de la scolarisation de la fiche de lecture permet de prendre conscience des processus de transposition didactique et de leurs enjeux. Support d'animation culturelle dans les mouvements d'éducation populaire d'après-guerre, la fiche de lecture traditionnelle apparaît dans les Instructions officielles dans les années 70 et se transforme souvent en exercice scolaire de pur contrôle bureaucratique des lectures personnelles des élèves, sans apprentissage progressif et sans réelle perspective de réinvestissement.

\section{Citer ce document / Cite this document :}

Privat Jean-Marie, Vinson Marie-Christine. La fiche de lecture ou la bureaucratisation d'une technique d'animation culturelle. In: Pratiques : linguistique, littérature, didactique, n90, 1996. Des méthodes en français. pp. 83-94; doi : https://doi.org/10.3406/prati.1996.1775

https://www.persee.fr/doc/prati_0338-2389_1996_num_90_1_1775 


\title{
LA FICHE DE LECTURE
}

\author{
ou la bureaucratisation d'une \\ technique d'animation culturelle
}

Jean-Marie PRIVAT

Marie-Christine VINSON

"Une histoire de l'enseignement est la meilleure des écoles pédagogiques » (1) $\mathrm{E}$. Durkheim

Selon une enquête récente menée auprès de professeurs de français, la fiche de lecture semble être, de loin, « la plus répandue » des modalités scolaires de rapport aux livres (2). L'enjeu est donc d'importance dans la mesure où la didactique de la littérature est en partie marquée par ce type de travail.

Notre réflexion naît d'un intérêt et d'un étonnement.

La connaissance de l'histoire culturelle ou institutionnelle d'un exercice scolaire, quel qu'il soit, est d'abord un gage de liberté intellectuelle, un garant d'autonomie professionnelle, un facteur de créativité didactique : sans ce type de «culture pédagogique » rappelle Durkheim, routine et immobilisme menacent gravement la pratique d'enseignement (3).

D'autre part, l'enseignant, même débutant, n'est pas sommé en principe d'accepter l'héritage pédagogique sans recul critique. Or, la fiche de lecture

(1) E. Durkheim, L'évolution pédagogique en France, (cours professés par le sociologue historien à partir des années 1904-1905 et repris jusqu'à la guerre ; 1re édition en 1938), P.U.F., Quadrige, Paris, 1990, Chapitre Premier, "L'histoire de l'enseignement secondaire en France - Intérêt pédagogique de la question » pp. 9-21.

(2) D. MANESSE et I. GRELLET, La littérature au collège, INRP/Nathan pédagogie, Perspectives didactiques, Paris, 1994, p. 67.

(3) E. Durkheim, id. On se reportera également aux travaux pionniers de Jean HÉBRARD - "La scolarisation des savoirs élémentaires à l'époque moderne ", pp. 7-58 - et de André CHERVEL - « L'histoire des disciplines scolaires : réflexion sur un domaine de recherches », pp. 59-121 - publiés sous la direction de Pierre Caspard dans le ${ }^{\circ} 38$, mai 1988, Pour une histoire des disciplines scolaires, de la revue du Service d'histoire de l'éducation de l'I.N.R.P., Histoire de l'éducation. 
semble bien faire partie de ces pratiques scolaires tenues pour naturelles, évidentes, légitimes en un mot... (4)

Notre hypothèse est que l'aspect indécis, hétéroclite et sommaire des attentes ou des visées de l'institution scolaire en la matière est le témoin symptomatique de la surprenante histoire de la scolarisation de la fiche de lecture. En toute hypothèse, cette histoire nous paraît fort instructive de l'orientation bureaucratique donnée, dans les faits, à la fiche de lecture.

La fiche de lecture est née dans les années d'après-guerre des préoccupations culturelles et sociales des mouvements d'éducation populaire et permanente. Lumière portée de la Résistance intérieure, «Peuple et Culture ", «premier mouvement français de formation d'animateurs » (5), s'est particulièrement intéressé à résoudre les problèmes de médiation culturelle. Deux principes organisent son action. D'une part, la lecture ne doit plus être considérée comme le supplément d'âme qui conviendrait à quelques esprits d'élite et à quelques enjeux académiques mais comme " une attitude active enrichissant à la fois sa personnalité, ses connaissances et son action ». D'autre part, il est réaliste de considérer que toute une pédagogie est nécessaire (panneaux, expositions, guides de lecture, clubs de lecture, cycles culturels, etc.) pour favoriser le contact entre les livres et leurs futurs lecteurs. C'est précisément pour aider le lecteur à apprécier, comprendre, comparer, juger, afin d'agir que sont mis en forme les premiers modèles de fiche de lecture (6) proposés aux " orienteurs culturels", les médiateurs culturels de l'époque.

(4) Ce phénomène n'est en rien exceptionnel dans la mesure où " la transposition didactique est ordinairement opaque [...]. Au terme du processus de transposition, l'objet d'enseignement apparaît dans une sorte de transparence originelle : l'effet terminal d'évidence, indispensable : quelle crédibilité sociale aurait un savoir entaché de manipulations apparentes ? repose sur l'occultation du processus " (J.-F. HALTÉ, La didactique du français, Paris, P.U.F., 1992, p. 51). C'est très exactement le dévoilement et le résultat du "processus" qui est l'objet de notre article.

(5) B. CACÉRÉS, Histoire de l'éducation populaire, Éditions du Seuil, Paris, 1964, p. 157

"'Peuple et Culture' (PEC) est né pendant la guerre, de la rencontre de gens qui partageaient les mêmes valeurs mais possédaient des cultures et des savoirs différents ; entre 1940 et 1944, dans les Alpes, des 'équipes volantes', dont les membres sont formés à l'école des cadres d'Uriage, sont en contact avec les maquis. Dès la libération de Grenoble en 1944, elles mettent sur pied des institutions d'éducation populaire, qui deviennent PEC en 1945.

L'organisation de stages, la fondation de revues au cours des années 1946-1947 [...] s'accompagnent de fiches destinées au club de lecture " (cité d'après Y. ALIX et M.-P. SCHMITT, "Les mouvements associatifs et militants ", Histoire des bibliothèques françaises, Les bibliothèques au XX siècle (1914-1990), sous la dir. de M. POULAIN, Promodis-Editions du Cercle de la Librairie, Paris, 1992, p. 320).

(6) G. CACÉRĖS, Regards neufs sur la lecture, en collaboration avec M. THOMAS, M. CITRON, J. DUMAZEDIER, R. GAUVIN, F. LAPORTE, P. RIVENC, P. ROYER, A. BAZIN, coll. Peuple et Culture, Paris, Le Seuil, 1949 (rééd. augmentée et actualisée en 1961, avec la collaboration de J. DUMAZEDIER, G. JEAN, J. HASSENFORDER)

Les fiches de lecture font l'objet du chapitre $V$ de la deuxième partie de l'ouvrage consacré à « La bibliothèque et les lecteurs" (suivie d'une partie "Documents " qui présente des exemples de fiche et quelques propositions de montages).

Les auteurs distinguent la fiche grand modèle et la fiche petit modèle ; l'une et l'autre " ne prétendent pas autre chose qu'à essayer d'apporter les éléments nécessaires pour bâtir un panneau de présentation de livres autour d'un thème, confectionner un guide de lecture, animer un Club de Lecture. Elle ne doivent supprimer ni la lecture du livre, ni la recherche. Offrant sur l'oeuvre tous les renseignements pédagogiques et culturels qui peuvent la mettre en valeur, elles sont là seulement pour aider les animateurs qui veulent faire de leur bibliothèque un véritable foyer de culture [...]. Les fiches serviront encore au bibliothécaire soucieux de confectionner les fiches illustrées de livres mises à la disposition des lecteurs » (op. cit., p. 139).

Ces fiches - publiées mensuellement - entrent dans les méthodes d'éducation par le livre, d'initiation vivante à la culture par le livre (entre autres). Elles sont utilisées dans les Cercles d'éducation populaire, les Amicales laïques, les Cercles de jeunes, etc. et déjà à la fin des années 50 dans « les classes terminales des Collèges d'Enseignement général, les Écoles Normales, les Centres d'apprentissage " (op. cit., p. 140).

Elles sont l'objet d'un travail très approfondi : elles sont adaptées à chaque ouvrage et à chaque public, soumises si possible à la critique de l'auteur, modifiées en fonction des remarques formulées par les utilisateurs, réélaborées aux termes d'une étude entre P.E.C. et I'Union italienne de la Culture populaire (G. Cacérés, op. cit., pp. 133-134). 
Or, en 1961, dans un article fondateur publié dans L'Éducation nationale (7), Georges Jean, professeur d'école normale et membre de PEC estime que pour lutter contre ce que l'on appelle " la crise du français à tous les niveaux de l'enseignement [...] cet instrument de travail mis au point dans le domaine de l'éducation populaire peut contribuer à redonner de la vie aux méthodes traditionnelles de l'enseignement littéraire ". Autrement dit, faciliter la rencontre avec la culture et ses enjeux ( « sans didactisme ou facilité ") est l'ambition humaniste et pédagogique du principe de la fiche.

\begin{abstract}
"Chaque fiche comporte deux parties principales :La première partie comprend une série de rubriques concernant l'œuvre et l'auteur envisagés. Après avoir indiqué le lieu, le milieu social, l'époque où se déroule le roman, la fiche présente un bref résumé de l'action et analyse en les distinguant soigneusement les thèmes du livre, les thèses qui s'y trouvent défendues, le 'point de vue' auquel se place l'auteur. Les questions concernant la forme, où l'on distingue le ton, le mouvement, le langage sont envisagées ensuite, ainsi que les problèmes que l'œuvre pose aux hommes d'aujourd'hui. Les pages présentant un intérêt documentaires sont soulignées. Une biographie de l'auteur et une bibliographie le concernant complètent cette première partie où l'on aborde parfois, et de plus en plus, des problèmes littéraires plus précis [...].

II va sans dire que l'organisation de la fiche varie beaucoup d'un ouvrage à l'autre. Contrairement à nombre de manuels scolaires ou de critique où l'on applique les mêmes schémas d'analyse à des ouvrages très différents, les fiches de lecture s'efforcent de faire surgir l'appareil critique des structures mêmes de l'ouvrage envisagé.

La seconde partie de la fiche est consacrée à la présentation d'un montage dont la durée excède rarement une heure [...]. Le montage d'un roman n'est ni un 'digest' ni une suite de morceaux choisis. II s'agit d'un découpage conçu pour qu'un animateur puisse communiquer à haute voix l'essentiel d'une œuvre, sa dynamique intime et profonde. Les passages qu'on lit sont reliés entre eux par des transitions organiques. Soucieuse de ne pas trahir l'œuvre, la méthode vise à en exprimer la substance [...]. Des notes pour la discussion suivent ce montage qui est précédé de quelques recommandations concernant la lecture expressive à haute voix ».
\end{abstract}

On comprend que la structure de la fiche de lecture en fait un « outil permettant à un animateur de tenir un club de lecture. La lecture du montage sensibilise l'auditoire, puis la discussion permet de passer des impressions aux idées, et des idées à certaines prises de conscience. Pour conduire sa discussion, l'animateur a besoin de trouver dans un document élaboré les renseignements précis et simples directement utilisables pour aiguiser l'appétit de son auditoire, inciter chacun à lire pour soi seul le livre présenté, et d'autres livres du même auteur. II ne s'agit pas de supprimer la lecture individuelle et silencieuse, mais de la susciter et de la féconder, la fiche étant beaucoup plus un moyen qu'une fin " (8).

G. Jean poursuit sa défense et illustration de la fiche de lecture en assurant que cette médiation chaleureuse et structurée induit une communion culturelle et émotive : «Elles ont permis à certains d'entre nous de sentir lors des veillées en milieu populaire que l'étincelle jaillissait, que le sang circulait à nouveau, que

(7) G. JEAN, "Fiches de lecture », Rubrique Techniques de la classe, L'Éducation nationale (publication du Comité universitaire d'Information pédagogique), n 11, 16 mars 1961, pp. 19-20.

Sur L'Éducation nationale comme revue " officieuse " du Ministère de l'éducation (de sa création en 1946 au début des années 70), se reporter à J. HÉBRARD et A.-M. CHARTIER, Discours sur la lecture, (1880-1980), Études et recherche, B.P.I., Centre Georges-Pompidou, Paris, 1989, pp. 309-312.

(8) G. JEAN, op.cit. L'auteur n'exclut pas que la fiche puisse constituer " une base de documentation solide pour l'élève ". 
Balzac, Sthendal ou Camus revivaient vraiment dans les yeux et dans les cœurs des paysans et des ouvriers qui découvraient dans une salle de café ou une cantine d'usine que la littérature est parfois beaucoup plus que la littérature ". Et de s'interroger rhétoriquement : "Pourquoi serait-il impossible de retrouver ce miracle dans nos classes ? » (9)

Utopie populiste et pédagogique ? Le miracle n'a pas eu lieu (10). Pour bien comprendre les formes dominantes et les enjeux de l'usage scolaire de la fiche de lecture, il n'est pas inutile de revenir rapidement sur les différents types ou prototypes qui l'ont précédée.

Au début de ce siècle, E. Faguet conseillait d'établir des fiches de lecture au lecteur qui voulait « lire en critique »(11) :

«II faudra lire lentement en prenant des notes et même en notant sur fiches. Fiches relatives à l'invention, aux idées nouvelles ; fiches relatives à la disposition, au plan, à la manière dont l'auteur conduit ses idées ou conduit son récit, ou mêle ses idées à son récit ; fiches sur le style, sur la langue ; fiches de discussion enfin, c'est-à-dire sur les idées de l'auteur comparées aux vôtres, sur son goût comparé à celui que vous avez, sur ses idées encore et son goût comparés à ceux de notre génération ou à ceux de la génération dont il était, etc.

De toutes ces fiches, vous constituez l'idée générale que vous vous faites de l'auteur et les idées particulières que vous avez sur lui et vous n'avez plus qu'à rattacher logiquement ou vraisemblablement ces idées particulières à cette idée générale, pour faire, sinon un bon article, du moins un article qui se tienne ».

II s'agit donc clairement de lire ici en professionnel et non de "lire pour jouir de sa lecture ", étant entendu que les situations et les enjeux de ce travail de lecture sont de l'ordre de la médiation littéraire écrite. Faguet résume le processus de communication en ces termes : "La lecture se compose de trois personnages : l'auteur, le lecteur ; et le critique est le médiateur ».

Entre cette fiche du lecteur professionnel classique (à la Faguet) et la version académisée de la fiche de l'animateur culturel (style Peuple et Culture), on peut repérer dans les pratiques d'enseignement et les Instructions Officielles une forme scolaire intermédiaire de médiation : "le compte rendu oral de lecture ".

"Les comptes rendus de lecture sont prescrits à partir de la cinquième et de la première année d'école primaire supérieure [...]. Ces lectures pourront être empruntées soit aux textes inscrits au programme, soit aux ouvrages de la bibliothèque de la classe ou de l'établissement. L'élève chargé du compte rendu n'aura

(9) Id., ibid

(10) Dans l'esprit des promoteurs de la lecture populaire, l'École bénéficie d'une structure pédagogique qui lui permet d'assurer sa mission de formation d'éducation ; c'est pour s'assurer d'un réel impact sur la formation des esprits que Peuple et Culture invente des dispositifs nouveaux et spécifiques... qui seront une vingtaine d'années plus tard scolarisés pour lutter contre la crise de l'enseignement de la littérature et de la lecture à l'École : “A l'école primaire, au collège, l'instituteur et le professeur disposent de la composition française ou de l'explication de textes pour essayer de former les esprits à un art de penser et de s'exprimer : au Foyer, à l'Amicale, à la Maison des Jeunes de quels moyens directs, sans le Club de lecture, disposerait l'animateur ? (G. CACÉRÉS et alii, op. cit., p. 130).

(11) E. FAGUET, L'art de lire, Paris, Hachette, 1911, p. II (rééd. Paris, Armand Colin, coll. L'Ancien et le Nouveau, 1992, pp. 6-7).

En 1971, dans son Dictionnaire de la langue pédagogique (Paris, P.U.F., p. 217), Paul FOULQUIÉ envisage encore la fonction les fiches de lecture selon un mode traditionnel : " feuilles ou cartons légers de format uniforme $(12,5 \times 20)$ sur lesquels on note, en vue d'une utilisation prochaine ou personnelle, soit le résumé d'un ouvrage qu'on vient de lire, soit une analyse méthodique accompagnée de réflexions personnelles, soit enfin des extraits textuels ". 
pas nécessairement à résumer un ouvrage, mais pourra s'en tenir à un épisode caractéristique. Le professeur veillera à ce qu'il ne se borne pas à lire une analyse écrite, mais à ce qu'il improvise, au moins pour une bonne part, son exposé, en n'utilisant qu'un plan et quelques notes [...]. II importe que les élèves pratiquent régulièrement ces compte rendus [...]. Des exercices de conversation et de discussion dirigée [...] peuvent faire suite au compte rendu de lecture qui servira de thème à la conversation et à la discussion »(12).

Ce type de compte rendu oral, à partir de notes de lecture et suivi d'une forme d'échanges dans la classe, est encouragé dés les années 30 ; il se maintient dans les programmes au moins jusqu'en 1976 dans la rédaction immuable suivante qui cependant met davantage l'accent sur la qualité de la communication orale que sur la discussion qui peut suivre :

« Lectures faites hors de la classe et donnant lieu à de brefs comptes rendus ou à des interrogations habituant les élèves à s'exprimer avec correction et aisance » (13).

La première occurrence officielle de la fiche de lecture concernant le lecteur scolaire date, à notre connaissance, des Instructions de 1977 pour les classes de français de $6^{\mathrm{e}}$ et $5^{\mathrm{e}}$ des collèges. Les élèves doivent apprendre, entre autres, à rédiger des « documents d'intérêt pratique et des comptes rendus écrits qui permettent, en liaison avec des activités ou travaux de la classe, d'initier aux problèmes que posent l'organisation et le ton de certains types de rédaction ». Ainsi - et le législateur de l'époque se doit d'expliciter les règles de composition et d'usage de ce nouvel exercice - " une fiche de lecture présente, après l'indication de l'auteur et du titre, un résumé de l'ouvrage rédigé en termes neutres ; elle peut comporter, en outre, selon la destination prévue, une appréciation personnelle » (14).

Les mêmes instructions précisent quelques pages plus loin, sous la rubrique lecture cette fois, que «quel que soit leur objet (œuvre proposée par le professeur ou par les élèves, avec, notamment l'appui du centre de documentation et d'information), quelle que soit la destination prioritaire qui leur est assignée (constitution d'un répertoire, entraînement aux méthodes d'organisation ou à la rédaction) ", fiches de lecture, résumés de lecture ou comptes rendus de lecture " ont pour résultat d'augmenter en le précisant le profit tiré de la lecture. A ceux qui en sont chargés, ils imposent, pour la mise en forme d'une communication orale ou écrite, une mobilisation - encore modeste - des facultés d'analyse et de synthèse ; ils permettent un engagement personnel. Ils incitent ceux qui ne connaissent pas les textes à les découvrir pour leur propre compte. A tous, ils offrent un enrichissement intellectuel, affectif, esthétique et moral »(15).

(12) Instructions relatives à l'application des arrêtés du 30 août 1937 et du 11 avril 1938 fixant les programmes de l'Enseignement du second degré (enseignement secondaire et enseignement primaire supérieur).

Sur les comptes rendus de lecture comme prolongement ou complément des lecture faites en classe, voir le Memento à l'usage des professeurs et élèves-professeurs de lettres et grammaire (Sevpen, 1964). Le professeur oriente la curiosité des élèves en classe, étant entendu que l'interrogation tout en servant de contrôle, pourra augmenter notablement le profit tiré de la lecture. L'enseignant pourra charger un élève d'un compte rendu oral de cinq ou dix minutes à l'adresse de ses camarades, suivi d'un entretien dirigé.

(13) Voir par exemple, Programmes 1938, Enseignement du second degré, $1^{\circ}$ cycle, Paris, Librairie Hachette, 1938 , pp. 53, 55, 57 et Deuxième cycle de l'enseignement du second degré, Horaires-Programmes-Admission dans les classes, $1^{\text {re }}$ édition, 1972, Paris, Programmes Vuibert, p. 15.

(14) Classes de $6^{e}$ et $5^{e}$ des collèges, horaires,objectifs, programmes et instructions, français, Programmes Vuibert, Paris, 1977, p. 37.

(15) Id., ibid., pp. 48-49. 
Les Instructions de 1979 - applicables aux classes de $4^{\mathrm{e}}$ et de $3^{\mathrm{e}}$ - considèrent à leur tour que " l'appropriation d'une culture accordée à la société de notre temps " passe entre autres par des travaux qui visent à " dégager méthodiquement d'un texte les apports les plus significatifs ». C'est le cas par exemple de l'établissement de « fiches de lecture qui, répondant à des questions déterminées, sont susceptibles de prendre place dans un répertoire utilisable par la classe ou d'être recueillies au centre de documentation et d'information, rendant le recours aux bibliothèques plus fructueux » (16).

Suivent d'autres propositions d'exercices (comptes rendus oraux ou écrits, exposés, discussions, débats, suites de textes, transpositions, adaptations, réflexions critiques) qui sont considérés comme marqués par un " degré supérieur d'exigence et d'efficacité » voire porteurs d'une vertu « plus formatrice » (17).

Les programmes actuels de français pour les collèges, en vigueur depuis 1985 , font référence à plusieurs reprises à la fiche de lecture comme exercice de rédaction qui " force le rédacteur à s'impliquer dans une activité de communication » (18), comme exercice de " développement des capacités d'abstraction et de raisonnement logique " (19) ou comme activité de lecture quand la fiche est l'expression d'une " lecture personnelle » (20) ou quand la constitution de fiches de lecture est " destinée notamment à un fichier spécial du CDI 》 (21).

Les Instructions et Programmes pour les classes de seconde recommandent la constitution de fichiers pour le développement de méthodes de travail personnel " efficace » (22) et proposent la fiche de lecture comme exercice de rédaction de " documents pratiques " à introduire dans une situation de communication (23).

En classe de première, l'étude d'une œuvre intégrale «peut donner lieu à l'élaboration de fiches individuelles ou collectives " à moins que le professeur ne se contente de suggérer la mise " au net d'une fiche », l'objectif étant plutôt dans ce cas d'aider l'élève à améliorer sa " maîtrise technique » dans des exercices préparatoires aux sujets d'examen (24).

La fiche de lecture est donc dans l'esprit du législateur un exercice parmi d'autres mais de plus en plus affirmé, à partir de la $6^{\mathrm{e}}$. Cette présence semble s'imposer comme allant de soi au fil des instructions alors même que les vertus

(16) Objectifs, programmes et instructions, Paris, Vuibert, 1979 , p. 34

On retrouve la même formulation dans les Instructions de 1981 pour la Classe de Seconde des lycées. La fiche de lecture figure à la rubrique "Activités liées à l'étude des textes " parmi les "Travaux écrits divers " (avec les dossiers, monographies, transpositions discursives, adaptations diverses) ; la fiche est distinguée des "notes de lecture " qui précisent, " pour les fixer, des impressions personnelles " (Programmes Vuibert, Paris, 1981, p. 43)

Ces travaux ont pour objet " d'étendre et d'approfondir des connaissances, de former aux méthodes de réflexion et d'organisation, par-là, de favoriser des comportements de décision. Ils entraînent à la pratique de la communication et de l'expression : précision du vocabulaire, correction de la syntaxe, respect de l'orthographe, netteté de la présentation. A ces titres, ils ont une grande portée " (Id., ibid.).

(17) Id. ibid.

(18) M.E.N., D.L.C., Français, Langues anciennes, classes des collèges, $6^{\circ}, 5^{\circ}, 4^{\circ}, 3^{\circ}$, Horaires / Objectifs / Programmes / Instructions, CNDP, Paris, 1993., p. 37 et p. 54.

(19) Id., p. 56

(20) Ibid., complément Lire au collège (1986), p. 70.

(21) Le législateur explique qu'un même livre objet de plusieurs fiches peut avoir pour utilité de " faire prendre conscience de la diversité des points de vue " (ibid., p. 72).

(22) M.E.N., D.L.C., Français, Classes de seconde, première et terminale, Horaires / Objectifs / Programmes / Instructions, CNDP, Paris, 1992, p. 48.

(23) Id., ibid., p. 52.

(24) Id., ibid., p. 60 et p. 64 
de ce mode de rapport à la littérature sont à la fois, on l'aura noté, fort éclectiques et fort vagues. Les prescriptions pédagogiques officielles hésitent manifestement entre des objectifs rudimentaires plutôt centrés sur l'exercice de capacités cognitives ou sur la maîtrise de compétences d'écriture méthodiques et des enjeux culturels très généraux, surtout liés à l'exploitation plus rationnelle des lectures individuelles ou collectives.

Cependant cet éclectisme, qui procède sans doute d'une logique institutionnelle de cumul plus que d'un processus de réorganisation logique, présente de fait l'intérêt d'une ouverture - certes anarchique - sur des usages diversifiés. Or, les enseignants ne paraissent pas tirer souvent profit de cette situation de relative liberté didactique : la pratique la plus répandue dans les classes réduit ce bric-à-brac du discours de l'institution à un pur et simple exercice académique, normatif et standardisé.

Voici, à titre d'exemples, deux modèles ordinaires de fiches de lecture, le premier distribué aux élèves par leur professeur en collège, le second proposé par un manuel de français pour les lycées.

FICHE DE LECTURE 1 (collège)

TITRE

AUTEUR

ÉDITEUR

COLLECTION

COPYRIGHT (année de $1^{\text {re }}$ publication et dernière date de parution dans la collection)

GENRE (roman, conte, nouvelle, plusieurs récits...)

RÉSUMÉ (entre 100 et 120 mots, au présent)

PERSONNAGE PRINCIPAL (présentation -environ 100 mots)

PERSONNAGES SECONDAIRES (en énumérer entre 3 et 5 )

$\operatorname{LIEU}(\mathrm{X})$

ÉPOQUE(S)

\section{AU CHOIX :}

— plan de l'histoire

- milieu social (agriculteurs, artisans, oisifs...)

- précisez le genre par un de ces mots : sentimental, policier, fantastique, historique, psychologique, biographique, autobiographique, d'aventure...)

— précisez le ton employé (ou les tons) : poétique, comique, familier, ironique...

— qualifiez le livre : ex. il fait réfléchir - il est instructif - amusant..

- proposez un autre titre

— dites (entre 60 et 100 mots) ce qui vous paraît faire l'intérêt du livre

- biographie de l'auteur (ainsi que ses autres ouvrages) 


\section{FICHE DE LECTURE 2 (lycée (25))}

"Faire des fiches vous permettra de conserver les apports de votre lecture, en facilitera l'assimilation. Mais ces fiches ne doivent pas être établies n'importe comment. Elles ne seront pour vous des outils commodes, des serviteurs efficaces de la mémoire que si vous respectez ces quelques règles:

- établir toutes vos fiches selon un format standard ;

- prévoir des en-tête permettant de les classer logiquement et de les retrouver aisément ;

— réduire les renseignement notés à quelques éléments essentiels ;

- adopter la présentation la plus claire possible.

Pratiquement, vous noterez sur des feuilles de brouillon au fur et à mesure de votre lecture de même que vous soulignerez ou cocherez au crayon dans le livre telle phrase ou tel paragraphe plus important (ou simplement plus beau !). Mais, l'œuvre achevée, vous regrouperez l'essentiel sur une fiche.

Choisissez des feuilles de petit format ou des bristols parfois qui trouveront place dans un classeur aisément maniable.

$[\ldots]$

On distingue sur une fiche :

- un en-tête comprenant

* une lettre-code pour le classement : conventionnellement on adopte l'initiale du nom de l'auteur, ou du terme désignant le thème pour un classement thématique ;

*le nom de l'auteur ou l'intitulé du sujet ;

*les références de l'ouvrage (titre, date de la première publication, édition utilisée, date de cette édition) ;

- un bandeau indiquant la nature de la fiche, la date de la réalisation ;

- un titre précisant le contenu de la fiche : synthèse critique ou résumé de l'œuvre, citations remarquables, remarques personnelles, etc.

- les renseignements constituant le contenu de la fiche regroupés clairement sous la forme d'un plan schéma avec titres et sous-titres Les données retenues seront évidemment exprimées en style télégraphique, en utilisant les signes conventionnels de la prise de notes. Suite possible au verso.

- des marges permettant de faire figurer : à gauche les références (parties, chapitres, pages ou actes, scènes, vers, etc.) des points notés ; à droite des rapprochements avec d'autres œuvres ou des renvois à des œuvres critiques, des textes théoriques de l'auteur, etc.

De semblables fiches permettent la préparation d'un exposé, fournissent

(25) E. Calais, R. Doucet, Organibac, organisation, méthode et savoir-faire dans les études, Français I Méthodes, chapitre 2 - se cultiver - section 1 - Lire, "établissez des fiches de lecture ", pp. 66-68, Paris, Magnard, 1984. 
les références pour un devoir. On se contente de fiches plus sommaires, aux dimensions plus réduites, lorsqu'il ne s'agit que de garder les références d'une lecture personnelle pour une exploitation simplement éventuelle.

$[\ldots]$

Vous voici armé pour tirer le meilleur parti de vos lectures. A vous maintenant de parcourir le chemin qui vous fera passer du lecteur au liseur (personne qui lit beaucoup) ».

Comparons ces deux types de fiches scolaires à celles proposées naguère par Peuple et Culture. Les différences voire les oppositions parmi leurs traits constitutifs sont nettes, nombreuses et fondamentales.

Dans l'espace scolaire, ce n'est plus un animateur qui utilise une fiche pour s'adresser à un groupe mais, à l'inverse, ce sont les élèves qui produisent une fiche pour le maître. Dans le premier cas la fiche était le support à une communication orale en direction du public, dans le second elle est un travail écrit destiné le plus souvent à la seule lecture du maître. Dans le cadre de l'éducation populaire, la partie d'information sur "l'œuvre et l'auteur » est complétée par une lecture à haute voix d'un assez long montage de textes, pour aboutir au moment fort de la discussion-débat où se forgent des convictions et s'arment des volontés. A l'École, la fiche n'est que rarement l'objet d'un exposé oral ou d'une mise à disposition dans la classe ou au CDI à l'usage des pairs ; autrement dit, à l'enjeu d'émancipation sociale et culturelle est substitué un protocole magistral de contrôle et de notation des lectures. C'est du moins ainsi que la plupart des élèves semblent considérer l'exercice, le réduisant de façon réaliste, dans la logique du dispositif, à sa seule valeur d'échange purement scolaire : «A part pour la note, la fiche, je m'en fiche!».

Cet académisme se remarque encore dans la rédaction de fiches aux questions fixées une fois pour toutes et dans le resserrement sur le corpus romanesque alors que Peuple et Culture s'efforçait d'adapter véritablement l'outil de travail à son objet et ne s'interdisait pas la poésie ou des rapprochements avec d'autres genres artistiques et littéraires (cinéma, théâtre, documentaires).

La configuration et l'usage scolaire réels de la fiche de lecture sont donc très éloignés de ce que proposait les modernistes des années 60. Comment expliquer ces avatars ? Faut-il regretter cette évolution?

Ces questions posent le problème plus général de la transposition didactique et particulièrement de l'articulation entre pratiques sociales et culturelles de référence et pratiques scolaires (26). On partage l'idée que la scolarisation des savoirs s'effectue au sein d'une " conjoncture générale » (27) qui structure le

(26) Sur ces questions, voir en particulier les ouvrages classiques de Y. CHEVALLARD, La transposition didactique, Grenoble, La pensée sauvage, 1985, de J.-L. MARTINAND, Connaître et transformer la matière, Peter Lang, Berne, 1986 et l'article de P. ARNAUD, "Contribution à une histoire des disciplines d'enseignement : Ia mise en forme scolaire de l'éducation physique ", Revue française de pédagogie, oct.-nov.-déc. 1989, pp. 29-34.

(27) J.-F. HALTÉ, op. cit., pp. 57-58. 
système éducatif. Des contraintes et des logiques didactiques internes sont aussi à prendre en compte.

On peut esquisser le cadre général en rappelant que la crise de l'enseignement de la littérature et des pratiques personnelles de lecture ont conduit fort normalement l'institution à rechercher des techniques pédagogiques nouvelles qui permettraient de construire pour un public scolaire élargi un rapport nouveau d'appropriation des textes. Ces données socio-scolaires sont elles-mêmes à situer dans un contexte culturel plus général. En effet, la fiche de lecture correspond très bien, dans son principe, à la forme scolaire de la "lecture extensive » moderne qu'ont décrit les historiens des pratiques lectorales (28). La qualité et la relative accessibilité marchande du livre de poche et l'incitation généralisée à multiplier les expériences personnelles de lecture expliquent que la fiche puisse être considérée comme un outil adapté à cette donne culturelle (29). L'idée que rédiger une fiche c'est à la fois élargir son horizon de références littéraires et apprendre à travailler avec méthode pour un profit à la fois personnel et scolaire renforce la légitimité de l'exercice. Il existe enfin la conscience diffuse, sans doute, que cette manière d'objectiver sa lecture conjugue une certaine vitalité libérale propre à l'animation culturelle en lecture publique (le lecteur serait gratifié) et une forme intéressante d'organisation des connaissances voire de structuration des apprentissages dont l'École est responsable (l'élève serait éduqué). Cette conjonction surdéterminerait ainsi les connotations favorables dont jouit la fiche auprès de nombreux enseignants qui saisiraient logiquement cette opportunité professionnelle.

Le fait est que la fiche s'inscrit aussi dans un contexte didactique qui la réduit trop souvent à un pensum, document ni utile ni agréable, « mort » et « mortel » en fait... pour les élèves comme pour l'enseignant.

Il paraît fort raisonnable que dans le cadre de l'École, de ses objectifs et de ses publics spécifiques, les élèves soient les rédacteurs de la fiche et non le maître-animateur. La lecture d'œuvres intégrales que suppose en principe la technique de la fiche est une autre qualité que l'on peut lui reconnaître également. Mais pour autant le dispositif a perdu toute dimension appropriative personnelle et socialisée pour n'être plus qu'un « devoir de lecture » (30).

Les rubriques de la fiche sont en général imposées par le maître (les traits constitutifs de la fiche pourraient fort bien être élaborés avec les élèves) et quand il y a choix possible (voir fiche 1), c'est encore l'enseignant qui décide des entrées à retenir, ou abandonne l'élève à une fausse liberté. Cette imposition est souvent ressentie comme arbitraire par les élèves qui n'ont d'autres solutions que de s'accommoder de cette situation typiquement transmissive et autoritaire.

II n'est pas exclu que la rédaction de fiches ait des effets incitatifs sur des jeunes lecteurs dont les dispositions culturelles socialement conditionnées les prédisposent à intérioriser l'ascétisme scolaire de cet exercice. Cependant, pour l'ensemble des lectorats juvéniles qui fréquentent l'École, la fiche permet difficilement de construire des compétences de lecture. Comme le déclare, pour le

(28) R. CHARTIER, " Du livre au lire ", in Pratiques de la lecture, sous la dir. de R. Chartier, en particulier " Les figures de la lecture ", Marseille, Rivages, 1985, pp. 69-79 (rééd. Paris, Payot-Rivages, 1993).

(29) Sur ce point voir par exemple, A.-M. CHARTIER et J. HÉBRARD, Discours sur la lecture (1880-1980), Études et recherche, B.P.I., Centre Georges-Pompidou, Paris, 1989, notamment les pp. 368-379.

(30) Voir J.-M. PRIVAT, "Socio-logiques des didactiques de la lecture ", in Didactique du français, état d'une discipline, sous la dir. de J.-L. Chiss, J. DAVID et Y. REUTER, Perspectives didactiques, Nathan / pédagogie, Paris, Nathan, 1995, pp. 133-153. 
déplorer, une collègue, "la fiche n'est pas un outil de formation, c'est une commodité de contrôle des lectures, ni plus ni moins ». En effet, ce type de travail ne fait que rarement l'objet d'une réflexion didactique (fiches à entrées variables inscrivant le travail dans une pédagogie différenciée, progression organisée au cours de l'année en fonction des renseignements que livrent les fiches sur les profils de lecteurs et les compétences de lecture, concertation tout au long du cursus et d'un cycle à l'autre, modalités de socialisation orale et écrite entre pairs, rédaction collective, fiches rédigées en alternance avec le professeur pour alléger la tâche de lecture de l'élève, fiches contradictoires rédigées par plusieurs lecteurs sur un même livre, fiches sur des ouvrages documentaires, diversifications des enjeux et des situations de communication, évaluation formative et réécriture de la fiche, fiche construite selon des items sélectionnés en vue d'une tâche d'écriture particulière, en vue de l'information d'un public spécifique, etc.).

L'orientation descriptive et normative donnée en règle générale à la fiche bloque par ailleurs tout réel investissement personnel du lecteur qui pourrait... afficher sa lecture. "L'avis personnel » du lecteur est très souvent rejeté en fin de fiche sous forme synthétique et sommaire ; le jeune lecteur est ainsi quasiment contraint à la langue de bois critique dans la mesure où la réponse aux rubriques précédentes le conduisent à reconnaître la légitimité du livre ou du moins son «intérêt » (voir fiche 1). La fiche traditionnelle est donc aussi désuète et dogmatique dans la mesure où elle tend à conformiser les lectures et à conformer les lecteurs : " tout se passe dans l'Éducation comme si la forme de sa mise en place technique s'était réalisée démesurément, en éliminant le contenu même qui l'a rendue possible et qui dés lors perd son utilité sociale ". Tout se passe comme si lire un texte "c'était le recevoir d'autrui sans y marquer sa place, sans le refaire » (31). On postulera au contraire que "la créativité du lecteur croît à mesure que décroît l'institution qui la contrôle » (32), on reconnaîtra que l'appropriation lectorale comme dialectique dynamique entre inventivité du lecteur et contraintes textuelles est " au cœur même des processus de réception » (33), on ne méconnaîtra pas enfin que le nouveau lecteur de l'âge électronique est virtuellement convié à devenir un lecteur particulièrement interactif et productif, « coauteur » (34).

La bureaucratisation du dispositif est donc achevée dans cette élision du lecteur dont l'interaction personnalisée viendrait compliquer la tâche du correcteur de fiches, dans la standardisation du protocole de questionnement des œuvres, dans l'évaluation sommaire des apprentissages textuels et culturels, dans l'absence massive de socialisation littéraire orale ou écrite entre pairs, dans la rareté enfin des situations fonctionnelles de réinvestissement (projets de lecture ou d'écriture).

(31) M. de CERTEAU, « Lire : un braconnage ", L'invention du quotidien, Arts de faire, 1, Paris, U.G.E., 10/18, 1980, pp. 279-296.

(32) M. de CERTEAU, op. cit.

(33) R. CHARTIER, "Textes, imprimés, lectures ", Lire en France aujourd'hui, sous la dir. de M. Poulain, coll. Bibliothèques, Ed. du Cercle de la Librairie, Paris, 1993, pp. 15-29.

(34) R. CHARTIER, "Lecteurs dans la longue durée, du codex à l'écran ". Histoires de la lecture, Un bilan des recherches, sous la dir. de R. Chartier, IMEC Éditions, Ed. de la Maison des Sciences de I'Homme, Paris, 1995, pp. 271-283.

L'historien résume en ces termes l'activité du lecteur de textes électroniques: « II peut soumettre le texte à de multiples opérations (il peut l'indexer, l'annoter, le copier, le démembrer, le recomposer, le déplacer, etc.) mais, plus encore, il peut en devenir le coauteur [...]. II se trouve en position de constituer un texte nouveau à partir de fragments librement découpés et assemblés [...], il peut construire à sa guise des ensembles textuels originaux dont l'existence, l'organisation et l'apparence même ne dépendent que de lui. II peut, à tout moment, intervenir sur les textes, les modifier, les récrire, les faire siens ». 
On retrouverait bien sûr ce type de critiques à propos de l'apprentissage traditionnel de l'orthographe par la dictée ou du développement de compétences rédactionnelles par la rédaction coutumière.

La recontextualisation scolaire de la fiche de lecture nous paraît donc un exemple particulièrement malheureux d' « inanimation » culturelle : la fiche fige. Cette modalité de scolarisation du rapport à la littérature correspond plus globalement à la " posture scolastique " du lector academicus qui conduit à " traiter le langage non comme un instrument mais comme un objet [...] d'analyse ", dans la logique de la gratuité esthétisante et de l'intérêt désintéressé quoique obligé, autrement dit dans «la neutralisation des fins pratiques » (35).

Ce point de vue nous importe doublement. D'abord parce que la généralisation de l'étude des œuvres intégrales a suscité l'apparition de nouveaux exercices " dont la fiche de lecture est l'exemple le plus généralisé dans les deux cycles » (36).

Ensuite parce que « les exercices de l'enseignement scolaire [...] ne laissent pas d'influer considérablement sur certaines pratiques culturelles » (37) hors l'école, dans la vie présente et future des lecteurs.

S'il est vrai que " le savoir enseigné suppose un processus de naturalisation qui lui confère l'évidence incontestable des choses naturelles » (38), la vigilance didactique est d'autant plus nécessaire. Proposer des analyses historiques et critiques de "savoirs scolaires, décrire les modalités et les principes de leur scolarisation constitue une tâche méthodologiquement décisive, propre à apporter une plus grande connaissance des phénomènes. Sur un plan plus immédiatement pratique, attirer l'attention, fût-ce de façon négative, c'est déjà agir. La prise de conscience des phénomènes de transposition, la connaissance progressive des problèmes qu'elle pose constituent des éléments précieux pour l'action didactique » (39).

Ils permettent de réinscrire les savoirs et les usages professionnels dans la triple histoire raisonnée des pratiques sociales, des savoirs enseignés et des conjonctures éducatives.

(35) P. BOURDIEU, "Le point de vue scolastique ", Raisons pratiques, Paris, Seuil, 1994, pp. $219-236$.

(36) D. MANESSE, article "Français ", Dictionnaire encyclopédique de l'éducation et de la formation, coll. " réf. ", sous la dir. de Ph. Champy et Ch. Etévé, Paris, Nathan, 1994, p. 458.

On rencontre des modèles plus ou moins subtils de fichage de ses lecture dans les productions parascolaires, dans les pratiques de l'école primaire, dans les programmes actuels pour les classes de B.E.P., etc.

(37) A. CHERVEL, op.cit., p. 118.

(38) Y. CHEVALLARD, op.cit., pp. 14-15.

(39) J.-F. HALTÉ, op.cit., p. 59. 